\title{
Visual summation and its relation to processing and memory'
}

An experiment is reported in which the interval between two presentations of the same item was svstematically varied. The results indicate summation occurs only while the two stimulations are in the neural mode of storage. No interaction of input is observed if one of the items has already been stored when the second arrives. Some implications of the relation of processing to memory are discussed.

Perception may be defined as the extraction of information from a stimulus. As such, a number of experiments have been carried out which have provided considerable knowledge about how this extraction is carried out and entered into permanent memory. These experiments have dealt with such questions as the duration of processing (e.g., Sperling, 1960) as well as the role of familiarity (e.g., Mewhort, 1967). Few perceptual experiments, however, have been directed at the question of how materials are stored in memory after processing has been completed. Because of some effects. such as the effect of familiarity that seems to be localized at the interface between processing and storage (Mewhort, Merikle, \& Bryden, 1968), it is important to consider how the materials are permanently stored in memory.

There are two major alternatives as to the way storage could occur: (1) each presentation of an item could be stored independently of all other representations of that item or (2) each presentation could be added to the existing representations of that item. One way to view this distinction is in terms of cell assemblies (Hebb, 1949) as a convenient conceptual framework. Within this context, given a presentation of an item, it could be stored in one of two ways: Under the additive storage hypothesis. there could be a single cell assembly that contained all representations of that item. Under the independence hypothesis, there would be a number of different cell assemblies, each of which contained a representation of that item.

While the independence notion tends to be more consistent with biochemical data (Russell, 1966), such a notion has been difficult to reconcile with behavioral data. One of the major reasons for this difficulty is that investigators have seldom been specific in terms of the kinds or categories of information that reach storage. Not only is there the identity of the item (e.g., the letter A) but there is also information about the context in which the item occurred. This context might be a group of other letters, a mathematical formula, or a prose sentence, and will include spatial information as well as other categories of information. Effectively, this means there are two major questions about storage: the first is related to what and how information is stored in a single trace, while the second question is related to associations between traces and the role of stimulus context. Although highly important, we are not going to deal with contextual information here.

A number of studies by Eriksen (e.g., Eriksen, 1966a) have provided more specific support for an independence notion. On a given trial, Eriksen (1966a) presented one of three letters (A, T, or $U$ ) either once or twice in succession. Single presentations were shown in either of the two tachistoscope channels, double presentations were shown with one letter in each channel. The letters were presented at the corners of an imaginary square of $1.25 \mathrm{deg}$ per side centered about the fixation point. Using accuracy on the single presentation as the base, he found that accuracy on the double presentations could be predicted by a modified version of the binomial distribution. The close fit of the binomial implies independence of the two presentations. If the two presentations were not independent, the binomial would underestimate accuracy on double presentations. The amount of improvement of the double presentation over the single implies that the $S$ behaved as though he had two independent opportunities to perceive the stimulus.

In all cases in which independence was observed, the stimuli were separated spatially, this separation being $1.25 \mathrm{deg}$ or more. Eriksen (1966a) has interpreted these data as indicating that the underlying neural activity is not correlated for independent presentations. His interpretation can be extended, however. Since accuracy is independent, this implies that processing is independent under these conditions. Furtherlinore, since processing must determine what is stored, storage should also be independent.

Although Eriksen has clearly shown independence of spatially separated presentations, there is little direct evidence for independence of temporally separated events. The masking data do provide some indication that temporal independence exists in addition to spatial independence. For example, in masking situations, little masking is observed if the interval between the test and masking stimuli is greater than $200 \mathrm{msec}$. However, while these data are more consistent with an independence notion, the generality of the independence notion has not been fully documented. Therefore, the present experiment is an attempt to gather some information in terms of temporal independence.

\section{Subjects}

\section{METHOD}

Eight Ss were used, all of them introductory psychology students at Lake Forest College; participation fulfilled a course requirement.

\section{Stimuli}

The letters $A, U, O$, and $Y$ were used as stimuli. The stimulus cards were prepared with Letraset Instant Lettering (Style 115) with one letter on each card. The letters were lined up on the cards so that they wauld superimpose one another in Fields 1 and 2. They were presented in the center of the viewing field.

\section{Procedure}

For each $S$ there were two $1-h$ sessions on 2 different days. The first day was used to assess threshold and also for practice. The absolute threshold was taken as the point at which an $S$ could correctly identify the letters $50 \%$ of the time. Thresholds were determined separately for Fields 1 and 2 to take into account any differences that might exist between the fields. After the duration threshold was determined, three-quarters of the threshold value for each field was used as the test duration for the stimuli. Nevertheless, the sum of the respective durations for Fields 1 and 2 would be of suprathreshold duration.

The stimuli were presented in a Scientific Prototype, 


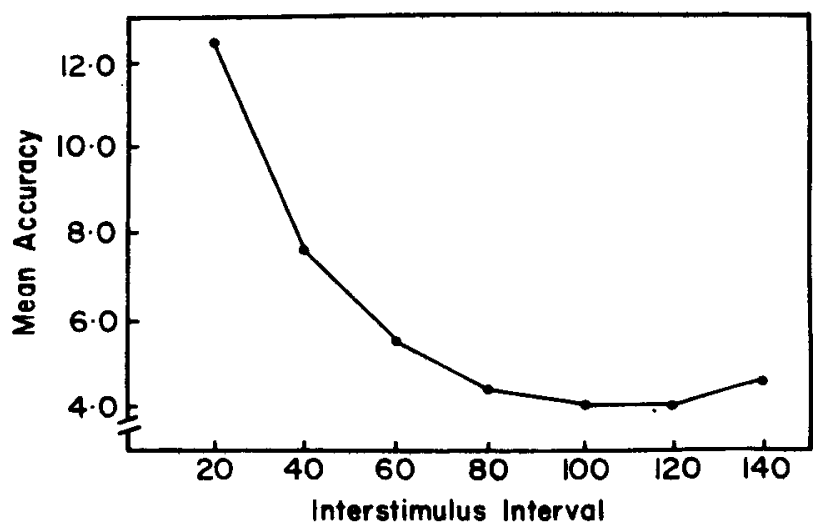

Fig. 1. Mean accuracy (maximum $=20$ ) for each interstimulus interval. Each point plotted represents a total of 160 trials over eight Ss.

three-field tachistiscope Model G. The letters were presented in Field 1 and Field 2 successively. The interstimulus in terval (ISI) was a blank field which also served as the illuminated preadapting field and postadapting field. So on a given trial the presentation sequence was as follows: Preadapting (blank), Field 1, ISI (blank), Field 2, postadapting (blank). Fields 1 and 2 remained on for a duration as determined for each $S$ and the ISI was systematically varied from 20 to $140 \mathrm{msec}$ in steps of $20 \mathrm{msec}$. The ISI was randomized in blocks of seven for each letter. Each of the four letters was shown five times at each of the seven durations from 20 to $140 \mathrm{msec}$, and therefore each S viewed 140 test presentations.

In addition, each $S$ was tested on each field individually. They were tested 10 times on each letter on both fields at three-fourths threshold, the same duration used for double presentation. This was done to provide baseline accuracy to compare double presentations with single presentations.

\section{RESULTS}

An analysis of variance for related measures (Ss by Duration by Letters) was applied to the data. The dependent variable was the number of correct responses for each condition; the scores used in the analysis were obtained by summing across repetitions of each condition. This analysis indicated that both Duration of the ISI $(p<.001)$ and Letters $(p<.05)$ produced significant effects. The interaction of Duration by Letters, however, was not significant $(F<1.00)$. The difference in the individual letter recognition is a result of differences in perceptability of individual letters, and since the interaction of Duration by Letters was not significant, the major effect of duration is not contaminated by this finding.

The overall effect of duration is shown in Fig. 1. To evaluate duration more precisely, the Duncan Multiple Range Test was applied to the means for each ISI (Edwards, 1968). This statistic indicated that the ISIs of 20,40 , and $60 \mathrm{msec}$ differed from each other and from the remaining intervals $(p<.01)$; however, none of the other comparisons approached significance. This suggests that asymptote was reached somewhere between 60 and $80 \mathrm{msec}$. The asymptote, however, does not indicate that summation is complete at that point.

To evaluate the possibility that summation occurred after asymptote, the results of the single presentations were used to generate predicted values assuming no summation. The probability of correctly identifying the letter on Field 1 alone was 0.13 and on Field 2 alone was 0.11 . These values were entered into the formula for independent probabilities of two events (Mosteller, Rourke, \& Thomas, 1961)

$$
P(A U B)=P(A)+P(B)-P(A \cap B) \text {. }
$$

Table 1 shows the predicted probabilities (assuming no summation) and the observed accuracy values from the double presentations. From the table it is quite clear that no summation occurs after asymptote and that after this point the presentations are handled independently.

One could ask about the role of guessing in this experiment. Past experience has indicated that in experiments of this type in which responses are not forced, Ss tend not to guess and in fact Mewhort (1967) has demonstrated experimentally that guessing does not play a major role in tachistoscopic recognition. Indeed, this was also the case in this experiment; of the responses emitted, only $4.8 \%$ were incorrect and therefore, most of the errors were of omission. Somewhat curiously, more than half of the incorrect responses occurred at ISIs of 20 or $40 \mathrm{msec}$, further indicating the effect of summation. As well as having appropriate or correct summation, no doubt there exists inappropriate summation. Inappropriate summation would result in errors and may reflect the operation of noise in the system.

\section{DISCUSSION}

The present experiment evaluated temporal summation in tachistoscopic recognition. The results indicated that accuracy of recognition was facilitated if two presentations of the same item were separated by less than $60 \mathrm{msec}$. For intervals greater than $60 \mathrm{msec}$, recognition accuracy was no better than would be predicted on the basis of no summation, i.e., independence.

A number of investigators have suggested that memory involves at least two phases: the first, a transient neural phase-the icon-(Neisser, 1967) and the second, a more permanent code (Hebb, 1949). Stimulus information must be transferred from the first into the second. The present data allow us to make some inferences about the way in which stimulus material is handled in the first phase and how it is coded in the second phase.

Sternberg (1966) has estimated that a letter requires $40 \mathrm{msec}$ to be processed. In the present experiment asymptote occurred after $60 \mathrm{msec}$ and since no summation occurred after that interval, the data suggest that roughly $60 \mathrm{msec}$ were required to process a single presentation in the present experimental situation. Although this estimate is at variance with Sternberg's, the two are not necessarily inconsistent. Neisser (1967) has suggested that the icon will vary as a function of energy variables; therefore any estimate of processing time will be a function of duration, luminance, and contrast ratios as well as the context of the task for $S$. In the present experiment there were no other stimuli to provide distinction although the duration of exposure was rather short making this task a difficult one. Thus the differences in estimates of processing duration can easily be understood in terms of the number of items $S$ is expected to identify and the ease with which he can identify a single item.

Whereas Eriksen (1966a) has shown spatially separated stimuli are processed independently in some cases, the present data suggest that temporally separated stimuli may also be processed independently. The importance of this effect lies in the implication about the way in which material may be stored

Table 1

Predicted Probabilities Generated from Single Presentation assuming no Summation and the Observed Probabilities from Double Presentations

\begin{tabular}{lccccccc}
\hline \multicolumn{7}{c}{ Duration of ISI } \\
\hline & 20 & 40 & 60 & 80 & 100 & 120 & 140 \\
\hline Predicted & .227 & .227 & .227 & .227 & .227 & .227 & .277 \\
Observed & .619 & .381 & .275 & .225 & .206 & .206 & .231 \\
\hline
\end{tabular}


permanently. Taken together, the spatial and temporal data suggest that what is contained in a permanent memory trace will be a function of the stimulating condition. In some cases two presentations of the same item or of different items may be encoded in the same trace or cell assembly. In other cases. however, two distinct traces may be established. (Of course, there is also the possibility of partially overlapping assemblies.) Both spatial and temporal information, however, are important in terms of context and one must be careful to distinguish between the storage of individual items and the connections between items. Unfortunately, a good deal more data will be necessary to make the distinction clearly between storage of contextual information and the storage of the item itself. At present, we do know that contextual information may or may not be useful depending on both the familiarity of the letter sequence and the spatial separation between items (Mewhort, 1966). No comparable data are available on sequential presentations. although the suggestion is that the familiarity effect should also change as a function of presentation rate.

As an alternative explanation of our results, Eriksen (1966b) has suggested as a possibility that as the interval between stimulations increases, more noise is added to the system. However, in the double-presentation experiment, such as this one, the only way noise can systematically influence perceptual accuracy is during the ISI, since $S$ responds immediately after the second presentation. Therefore, the longer the ISI, the greater the opportunity for noise to interfere with the processing of the first presentation. However, noise should have no systematic effect on the second presentation. Therefore, if noise is important, accuracy should continue to decrease to the level of the single presentation of Field 2, which is $11 \%$. Our results show that accuracy did not decrease to this level; therefore, there is no evidence that noise played a role in determining accuracy after asymptote. although the pattern of overt errors might be interpreted as indicating that noise plays a role prior to asymptote.

Our results do suggest that processing of form is not done in discrete time packages, or at least not in units in which the introduction of a second stimulation would interfere with the processing of the first. Instead, the data suggest that processing of form is continuous. Time is involved only inasmuch as there is a point in processing at which the transfer from iconic to more permanent storage must cease. The summation which occurs up until about $60 \mathrm{msec}$ is a result of the fact that the two stimulations are at least partially in the same mode of processing; i.e.. neural activity, and are thus more likely to summate. The amount of summation decreases with increases in ISI because the stimulation is moving further along in the neural mode. After stimulations have passed out of neural activity they go either into permanent storage or are lost from the system. The reason the accuracy curve asymptoted at $60 \mathrm{msec}$ may be that at greater intervals the first stimulation has already passed out of the neural mode before the second stimulation occurs. Therefore, the two stimulations will not summate once they are not in neural mode.

\section{REFERENCES}

FDWARDS, A. L. Ixperimental design in psychological research. 3rd ed. New York Holt, Rinehart, \& Winston, 1968.

r RIKSFN C. W. Independence of successive inputs and uncorrelated error in visual form perception. Journal of Fxperimental Psychology, $1966 \mathrm{a}, 72,26-35$.

FRIKSFN, C. W. Temporal luminance summation effects in backward and forward masking. Perception \& Psychophysics, 1966b, 1, 87-92. HEBB, D. O. The organization of hehavior. New York-Wiley, 1949.

MEWHORT, D. J. K. Sequential redundancy and letter spacing as determinants of tachistoscopic recognition. Canadian Journal of Psychology, 1966, 20, 435-444.

MEWHORT, D. J. K. Familiarity of letter sequences, response uncertainty, and the tachistoscopic recognition experiment. Canadian Journal of Psychology, 1967, 21, 309.321.

MEWHORT, D. J. K., MERIKLE, P. M., BRYDEN, M. P. On the transfer from iconic to short-term memory. Paper presented at Psychonomic Society, St. Louis, 1968.

MOSTELlER, F., ROURKE, R. E. K., THOMAS, G. B., JR. Probability and statistics. Reading, Mass.; Addison-Wesley, 1961.

NTISSER, U. Cognitive psychology. New York: Appleton-CenturyCrofts, 1967.

RUSSELL, R. W. Biochemical substrates of behavior. In R. W. Russell (Ed.), Frontiers in physiological psychology. New York: Academic Press, 1966.

SPERLING, $G$. The information available in brief visual presentations. Psychological Monographs General \& Applied, 1960, 74, (11, Whole No. 498).

STERNBFRG, S. High-speed scanning in human memory. Science, 1966 , $153,652-654$.

\section{NOTES}

1. This research was supported by the National Science Foundation, Grant GB-784R. to the second author.

2. Address. Department of Psychology, Lake Forest College, Lake Forest, lllinois 60045.

(Accented for publication Decemher 30, 1968.) 